\title{
Mean-field theory for Bose-Hubbard model under a magnetic field
}

\author{
M. Ö. Oktel, ${ }^{1, *}$ M. Niță, ${ }^{2}$ and B. Tanatar ${ }^{1}$ \\ ${ }^{1}$ Department of Physics, Bilkent University, 06800 Bilkent, Ankara, Turkey \\ ${ }^{2}$ Institute of Physics and Technology of Materials, P.O. Box MG7, Bucharest-Magurele, Romania \\ (Received 25 April 2006; revised manuscript received 16 August 2006; published 31 January 2007)
}

\begin{abstract}
We consider the superfluid-insulator transition for cold bosons under an effective magnetic field. We investigate how the applied magnetic field affects the Mott transition within mean-field theory and find that the critical hopping strength $(t / U)_{c}$ increases with the applied field. The increase in the critical hopping follows the bandwidth of the Hofstadter butterfly at the given value of the magnetic field. We also calculate the magnetization and superfluid density within mean-field theory.
\end{abstract}

DOI: 10.1103/PhysRevB.75.045133

PACS number(s): 67.40.Db, 05.30.Jp, 05.70.Fh

\section{INTRODUCTION}

One of the most interesting developments in ultracold atom physics is the study of neutral atoms in optical lattices. ${ }^{1}$ An optical lattice is prepared by creating a periodic potential utilizing a standing wave of light, and optical lattices in one, two, and three dimensions have been realized experimentally.

The cooling, trapping, and coherent manipulation of the atomic motion by their interaction with light has been established by numerous investigations in the field of atom interferometers, ${ }^{2}$ matter-wave superradiance, ${ }^{3}$ matter-wave parametric amplifiers, ${ }^{4,5}$ and others. ${ }^{6}$ One should mention also the opportunities garnered by using the ultracold alkali atoms as quantum computers, ${ }^{7}$ or by using the Mott insulating state of neutral bosonic atoms for detection of quantum entanglement. ${ }^{8-10}$ The custom-made trapping potentials in the optical lattice has also opened a venue to study many condensed matter problems, such as the Mott insulator transition experimentally realized by Greiner et al. ${ }^{11}$

Although many different regimes exist for optical lattice experiments, one that is quite interesting from a theoretical point of view is that of a deep lattice with few particles per lattice site. If fermions are used instead of bosons, these experiments may lead to direct realization of many correlated electron model Hamiltonians such as the Hubbard model of high-temperature superconductivity or lattice Quantum Hall models. $^{12}$

In this work, we concentrate on bosons, and assume that at each lattice site there is only one available state (that is equivalent to requiring the first excited state at each lattice site to be sufficiently high in energy). In this case the Hamiltonian is ${ }^{13}$

$$
\mathcal{H}=-t \sum_{\langle i j\rangle}\left(a_{i}^{\dagger} a_{j}+a_{j}^{\dagger} a_{i}\right)+\frac{U}{2} \sum_{i}\left(n_{i}-1\right) n_{i}-\mu \sum_{i} n_{i},
$$

where $a_{i}$ is the annihilation operator at site $i$ and $n_{i}=a_{i}^{\dagger} a_{i}$ is the number operator at site $i$. The first term corresponds to hopping between different lattice sites and for practical purposes only nearest-neighbor hopping is important, so the sum $\langle i j\rangle$ is carried over the nearest neighbors. The second term is the particle-particle interaction and the last term is the chemical potential. This is the widely studied Bose-Hubbard Hamiltonian. ${ }^{13-15}$
The strong tunneling limit between optical lattice sites $(U / t \ll 1)$ corresponds to the superfluid (SF) phase. Changing the laser intensity with increasing depth of the optical potential the atomic waves become more localized and the on-site interaction $U$ increases at the same time with the reduction of the tunneling parameter $t .{ }^{15}$ The system is driven to a Mott insulator (MI) phase and loses long-range phase coherence. In general, if the interaction is strong enough the system prefers a particle number that is commensurate with the number of lattice sites and the system goes into the insulating phase. The strength of interaction $U$ needed for this transition is roughly the bandwidth of the noninteracting system $2 z t$, where $z$ is the number of nearest neighbors.

A much less studied problem is that of the Bose-Hubbard Hamiltonian under a magnetic field. Experimentally, of course, the bosons used in cold gas experiments are uncharged and would not be directly affected by an external magnetic field. However, recent studies have shown in detail how a magnetic Hamiltonian, or, in general, effective electromagnetic fields, can be generated for atoms in optical lattices using an external time varying electric field ${ }^{16}$ by an oscillating quadrupole potential together with a periodic modulation of the tunneling between lattice sites ${ }^{17}$ or using more complicated laser configurations. ${ }^{18}$ These investigations suggest that it may be possible to study such systems with reasonable improvements on already functioning experiments. An effective magnetic field can also be created by rotating the optical lattice, and cancelling the centrifugal force of the rotation by an external quadratic trap. ${ }^{19}$ We also note the two recent papers which study how the artificial external non-Abelian gauge potentials can be created for cold atom systems. ${ }^{20,21}$

In this work, we assume that we have a two-dimensional square lattice in the $x-y$ plane, under a magnetic field in the $z$ direction. We also consider that the "charged bosons" are interacting only when they are on the same lattice site and the temperature of the system is set to zero. In this case the Hamiltonian is

$$
\begin{aligned}
\mathcal{H}= & -t \sum_{n, m}\left[a_{n m}^{\dagger} a_{(n+1) m}+e^{i 2 \pi \varphi n} a_{n m}^{\dagger} a_{n(m+1)}+\text { H.c. }\right] \\
& +\frac{U}{2} \sum_{n, m}\left(a_{n m}^{\dagger} a_{n m}-1\right) a_{n m}^{\dagger} a_{n m}-\mu \sum_{n, m} a_{n m}^{\dagger} a_{n m} .
\end{aligned}
$$


Here, we label every site of the lattice $i, j$ by two integers $i=\left(n_{i}, m_{i}\right)=(n, m)$, one integer $(n)$ along the $x$ axis, the other ( $m$ ) along the $y$ axis, and choose the gauge to be $\vec{A}=x \hat{y}$. The first term is the usual hopping term, where hopping along the $y$ axis gets a phase shift due to the presence of the magnetic field. Magnetic field affects the system through the parameter $\varphi$ with

$$
\varphi=B l^{2} / \varphi_{0},
$$

where $l$ is the lattice spacing and $\varphi_{0}$ is the flux quantum. Thus, the parameter $\varphi$ measures the magnetic flux per unit cell of the lattice in units of flux quantum. The second and third terms are interactions and chemical potential, respectively. Even at $U=0$, the noninteracting limit of this Hamiltonian shows interesting results; the energy spectrum at $U$ $=0$ is known as the Hofstadter butterfly. ${ }^{22}$ The most important aspect of the noninteracting problem is that the bandwidth depends critically on $\varphi$, and gaps open up or close in a self-similar manner. With such a complicated single particle spectrum, it is not at all clear how the presence of the magnetic field will change the Mott transition, or whether more exotic phases can be found.

We believe that with the possibility of experimental realization, it is of importance to study this model more closely and understand its rich phase diagram. In this work, we concentrate on the superfluid-insulator transition and investigate the effect of the external magnetic field on the phase boundary. Our mean-field approach is not capable of capturing possible correlated phases, however it may serve as a basis for more detailed investigation of the model.

We find that the Mott insulating phases become more stable under the applied magnetic field, an expected effect as one of the most important effects of the magnetic field would be to localize particles further. More importantly, we find that the critical hopping to interaction ratio $t / U$ roughly follows the bandwidth of the Hofstadter butterfly. We also calculate the magnetization and the superfluid density within mean-field theory.

In the rest of this paper we first outline our calculational scheme of solving the Bose-Hubbard model under a magnetic field within the mean-field approach. We then present our results on the phase diagram identifying the superfluid and insulating regions. We conclude with a brief summary of our main results.

\section{MEAN-FIELD APPROACH}

Our calculations are based on the mean-field approach of the Bose-Hubbard Hamiltonian, ${ }^{23}$ by considering the following decoupling formula for the product of the two Bose field operators:

$$
a_{n m}^{\dagger} a_{(n+1) m}=\left\langle a_{n m}^{\dagger}\right\rangle a_{(n+1) m}+a_{n m}^{\dagger}\left\langle a_{(n+1) m}\right\rangle-\left\langle a_{n m}^{\dagger}\right\rangle\left\langle a_{(n+1) m}\right\rangle .
$$

The average value $\left\langle a_{n m}^{\dagger}\right\rangle$ represents the order parameter $\Psi_{n m}$ that accounts for the insulator-superfluid transition. It is equal to zero on the insulator side of the transition when the ground state of the system has a definite particle number on every site of the lattice, and has a nonzero value for the superfluid state when there are large quantum fluctuations of the atom number in the optical lattice. In this case $\left|\Psi_{n m}\right|^{2}$ represents the local density of the atoms in the condensate state.

Using Eq. (5) the Bose-Hubbard Hamiltonian given in Eq. (2) turns into a sum of the following single-site terms:

$$
\begin{aligned}
\mathcal{H}_{n m}^{M F}= & -t\left[\Psi_{(n+1) m}^{*} a_{n m}^{\dagger}+\Psi_{(n-1) m}^{*} a_{n m}^{\dagger}+e^{i 2 \pi \varphi n} \Psi_{n(m+1)}^{*} a_{n m}^{\dagger}\right. \\
& \left.+e^{-i 2 \pi \varphi n} \Psi_{n(m-1)}^{*} a_{n m}^{\dagger}+\text { H.c. }\right]+U\left(n_{n m}-1\right) n_{n m}-\mu n_{n m} \\
& +C_{n m},
\end{aligned}
$$

where $n_{n m}$ is the single-site density operator $a_{n m}^{\dagger} a_{n m}$ and $C_{n m}$ is a constant energy term.

The matrix elements of the mean-field Hamiltonian $\mathcal{H}_{n m}^{M F}$ in the occupation number base $\left|N_{n m}\right\rangle$ are given by

$$
\begin{aligned}
\left\langle N_{n m}\left|\mathcal{H}_{n m}^{M F}\right| N_{n m}\right\rangle= & \frac{1}{2} U N_{n m}\left(N_{n m}-1\right)-\mu N_{n m}+C_{n m}, \\
\left\langle N_{n m}+1\left|\mathcal{H}_{n m}^{M F}\right| N_{n m}\right\rangle= & -t \sqrt{N_{n m}+1}\left[\Psi_{(n+1) m}^{*}+\Psi_{(n-1) m}^{*}\right. \\
& \left.+e^{i 2 \pi \varphi n} \Psi_{n(m+1)}^{*}+e^{-i 2 \pi \varphi n} \Psi_{n(m-1)}^{*}\right],
\end{aligned}
$$

where we used the property of the Bose field operators $c \mid N$ $+1\rangle=\sqrt{N+1}|N\rangle$ and $c^{\dagger}|N\rangle=\sqrt{N+1}|N+1\rangle$. All other matrix elements are zero, except the conjugate elements of Eq. (8). We note that the occupation number $N_{n m}$ above varies from 0 to $\infty$ and they are referred to the site $(\mathrm{nm})$ of the optical lattice. We diagonalize the Hamiltonian equation (6) in a truncated basis $\left|N_{n m}\right\rangle$ with $N_{n m}=0 \ldots N_{\max }$ and calculate the ground state of the mean-field Hamiltonian,

$$
\left|G^{n m}\right\rangle=\sum_{N=0}^{N_{\max }} \alpha_{N}^{n m}\left|N_{n m}\right\rangle
$$

with the coefficients $\alpha_{N}^{n m}$ corresponding to the lowest eigenvalue of the matrix of Eq. (6) in the truncated base.

The order parameter corresponding to the ground state given by Eq. (9) will be

$$
\Psi_{n m}=\left\langle G^{n m}\left|a_{n m}^{\dagger}\right| G^{n m}\right\rangle=\sum_{N=0}^{N_{\max }-1} \alpha_{N}^{n m^{*}} \alpha_{N+1}^{n m} \sqrt{N+1} .
$$

For a given truncated basis the equations of the finite Hermitian matrix in Eq. (7) and Eq. (8) and the formula for the SF order parameter, Eq. (10), represent a set of selfconsistent equations that give the solution of the ground state of the single site Hamiltonian, Eq. (6), and the order parameters $\Psi_{n m}$ in the mean-field approximation.

The numerical calculations are repeated with increasing values of the dimension $N_{\max }$ of the truncated basis to attain convergence of the solution. In the mean-field approximation, the ground state of the Bose-Hubbard Hamiltonian, Eq. (2), is given by the direct product of the single site ground states of Eq. (9),

$$
|G\rangle=\prod_{n m}\left|G^{n m}\right\rangle .
$$


For a given ground state, Eq. (9), the probability for the single site operator $n_{n m}$ to take the value $N$ will be given by the square of the corresponding developing coefficient $\alpha_{N}^{n m}$. The average single site occupation number denoted with $\rho(\mathrm{nm})$ is equal to

$$
\rho(n m)=\left\langle n_{n m}\right\rangle=\sum_{N=1}^{N_{\max }}\left|\alpha_{N}^{n m}\right|^{2} N,
$$

and the condensate component of the superfluid density, within mean-field theory, on the site $\mathrm{nm}$ is

$$
\rho_{s}(n m)=\left|\Psi_{n m}\right|^{2} .
$$

We denote by $\rho$ and $\rho_{s}$ the surface average of $\rho(\mathrm{nm})$ and $\rho_{s}(\mathrm{~nm})$, respectively. For a noninteracting system at zero temperature all of the bosons are in the condensate, i.e., in the lowest single particle state of the lattice, and we have $\rho$ $=\rho_{s}$. When the interaction increases [nonzero values of $U$ in Eq. (2)] only a significant fraction of the bosons will condense in the same single particle quantum state, and we have $\rho_{s}<\rho$. The competition between the kinetic energy of the system $t$ and interaction $U$ gives rise to interesting successive transitions between a superfluid and a Mott insulator.

The mean-field solution of the nonmagnetic system and the phase diagram are calculated by Sheshadri et al. ${ }^{23}$ See also the Mott insulator lobes in Figs. 1 and 2. For the magnetic Hamiltonian, perturbative techniques are used by $\mathrm{Ni}$ emeyer et al. ${ }^{24}$ where the MI lobes are calculated for small values of the magnetic flux $\varphi=0, \ldots, 0.125$.

Introducing the magnetic field in the hopping term of the Hamiltonian of Eq. (2) breaks the temporal invariance of the Hamiltonian and gives rise to persistent current flow of the "charged bosons." For any bond connecting neighboring sites $(i ; k)=(n, m ; n \pm 1, m)[$ or $(i ; k)=(n, m ; n, m \pm 1)]$ of the lattice, we define $t_{i k}=t$ for hopping along $x$ (or $t_{i k}=t e^{i 2 \pi n \varphi}$ for hopping along $y$ ). We calculate the local bond current of the superfluid phase using the following formula:

$$
v_{i k}=\frac{1}{i \hbar}\left[t_{i k} a_{i}^{\dagger} a_{k}-t_{k i} a_{k}^{\dagger} a_{i}\right],
$$

by substituting $\left\langle a_{i}^{\dagger} a_{k}\right\rangle$ with $\left\langle\Psi_{i} \Psi_{k}^{*}\right\rangle$. Another parameter of interest is the magnetic momentum. In the mean-field decoupling we can define the single site magnetization by the following formula:

$$
M_{n m}=n \bar{v}^{x}(n m)-m \bar{v}^{y}(n m)=-\frac{1}{\pi \hbar} \frac{d \Omega_{n m}}{d \phi},
$$

where $\Omega_{n m}$ is the average value of the mean-field Hamiltonian of Eq. (6) with respect to the ground state, Eq. (9), and represents the single site energy of the Bose gas. The averaged site velocities $\bar{v}^{x, y}(\mathrm{~nm})$ are equal to the average of the bond currents of Eq. (14) connecting the site $n, m$ to its neighbors along $x$ or $y$ accordingly. The magnetic momentum denoted with $M$ is equal to the surface average of Eq. (15).

For zero magnetic field the two-dimensional (2D) lattice has the translational invariance along both axes and the order parameter $\Psi_{n m}$ does not depend on the site index $(\mathrm{nm})$.
In our case, for nonzero magnetic field in the chosen Landau gauge the system preserves only the invariance along the $y$ axis of the lattice. Therefore, the order parameter is chosen as $\Psi_{n m}=\Psi_{n}$. In this case, we calculate the mean-field solution for a given ratio of the magnetic flux $\phi=p / q$. From the equation of the matrix elements of the mean-field equation (8), it can be noted that the periodicity of the solution is $\Psi_{n}=\Psi_{n+q}$. The same periodicity condition is verified by the the density $\rho$ that also shows the translational invariance along the $y$ axis: $\rho(n m)=\rho(n)$ and $\rho(n)=\rho(n+q)$. To solve the mean-field equations we choose a finite sequence of the lattice of dimension $q$ in $x$ direction and impose periodic boundary conditions. In the Landau gauge, the system is periodic with lattice periodicity in the $y$ direction, thus our calculations are carried out on a $1 \times q$ supercell with periodic boundary conditions. We measure the energy in units of $U$ (i.e., $U=1$ ).

\section{RESULTS AND DISCUSSION}

Based on our calculations outlined in the preceding section, we now present our numerical results. The two possible states of the 2D Bose system are selected as follows: in the MI phase, the on-site occupation number has integer values and the variance of $\rho$ is zero; in the SF phase the on-site occupation number has noninteger values and the variance $\sigma(\rho) \neq 0$. The main features of our results are illustrated in Figs. 1-6.

The phase diagram in the $\mu, t$ plane is depicted in Fig. 1, which is calculated for $\varphi=0$ and $\varphi=0.1$. For the Mott insulator state the site occupation number $\rho(\mathrm{nm})$ is equal to $\rho$ and has integer values (see Mott lobes in Fig. 1 for $\rho=1,2,3$ ). The variance of $\rho$ is equal to zero for the MI state and has large fluctuations for the SF state (see the variance of $\rho$ in Fig. 2).

The Bose-Hubbard model under a magnetic field has also been considered by Niemeyer, Freericks, and Monien, ${ }^{24}$ using strong coupling expansion. Strong coupling expansion utilizes a perturbative expansion in $t / U$, and is valid within the Mott insulating regime for small $t / U$ and small values of the flux $\varphi$. The mean-field approach of this paper, on the other hand, is a self-consistent but uncontrolled approximation, which makes it possible to calculate physical quantities for both the insulating and the superfluid regimes. Although the two approximation methods have very different character, they generally yield qualitatively similar results. Very similar phase diagrams have been obtained by these two different approaches for the pure Bose-Hubbard model (Refs. 23 and 25), and the superlattice Bose-Hubbard model (Refs. 26 and 27). Indeed, we find that our mean-field treatment produces a qualitatively similar result to the strong coupling expansion for the Bose-Hubbard model under a magnetic field. However, our method allows us to extend the calculation to the full range of magnetic flux $0<\varphi<1$, and calculate the superfluid density and magnetization in the superfluid phase.

We note that the transition point at zero magnetic flux is located at $t_{c}=0.043$ for $\mu=0.5$ (c.f. Fig. 1). This corresponds to $U / 4 t=5.8$ which is equal to the transition point determined by van Oosten et al. ${ }^{28}$ 

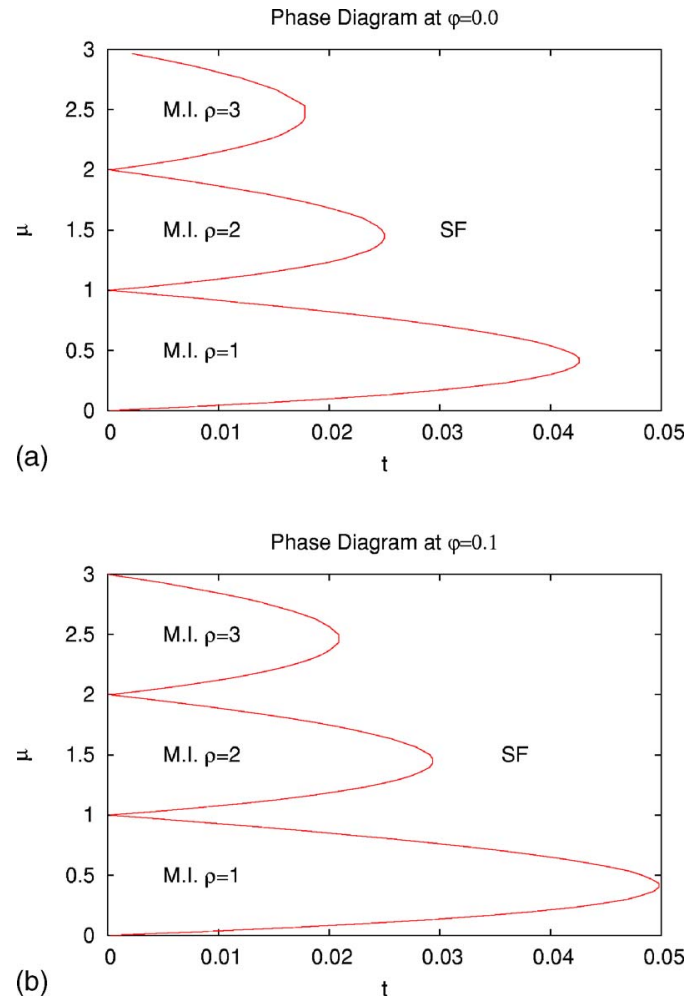

FIG. 1. (Color online) Phase diagram of Bose atoms confined in a 2D optical lattice for the magnetic flux $\varphi=0$ (left panel) and 0.1 (right panel). In the figure the first three Mott lobes are depicted, for on-site particle numbers 1,2 , and 3 .

The magnetic flux breaks the temporal invariance of the system and destroys the coherence of the wave function in a 2D fermion system after a "flight" time proportional ${ }^{29}$ to $1 / B$. For a Bose system, the magnetic flux can have the same effect over the SF coherent wave function, destroying the stability of the superfluid solution in the proximity of the transition point and leading to the SF-MI transition when $\varphi$ is bigger than a critical value $\varphi_{c}$. This is consistent with the increased area of the Mott lobes when the magnetic field is present as shown in Figs. 1 and 2. For all values of the magnetic flux we depicted the phase diagram in the $t, \varphi$ plane for the different values of the chemical potential in Fig. 3.

We note the above MI-SF transition for a constant $t$ value, increasing the magnetic flux $\varphi$ from zero to the critical transition point $\varphi_{c}$. For small $\varphi$ the optical lattice approximates a continuous system of charged bosons under the magnetic field and the superfluid-insulator transition is similar to the disappearance of the superconductivity when the external magnetic flux in a superconductor exceeds a critical value. ${ }^{30}$

However, for the Bose-Hubbard Hamiltonian the discreteness of the lattice brings about other interesting features. The noninteracting spectrum ${ }^{22}$ is periodic in $\varphi$ with $\Delta \varphi=1$ and symmetric around the value $\varphi=0.5$. We recover the same feature of the phase diagram in Fig. 3.

One of the most important results of our calculation is displayed in Fig. 3. The oscillations of the critical hopping strength with changing magnetic field follow the oscillations of the bandwidth of the Hofstadter butterfly ${ }^{22}$ (see Fig. 4). For instance, increasing $\varphi$ causes the bandwidth of the Hof-
Phase Diagram at $\varphi=0.0$

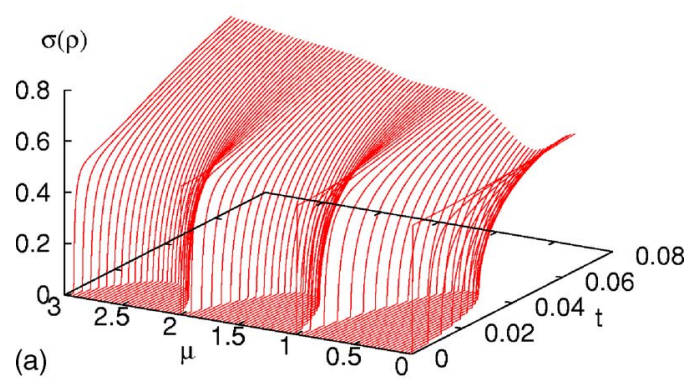

Phase Diagram at $\varphi=0.4$

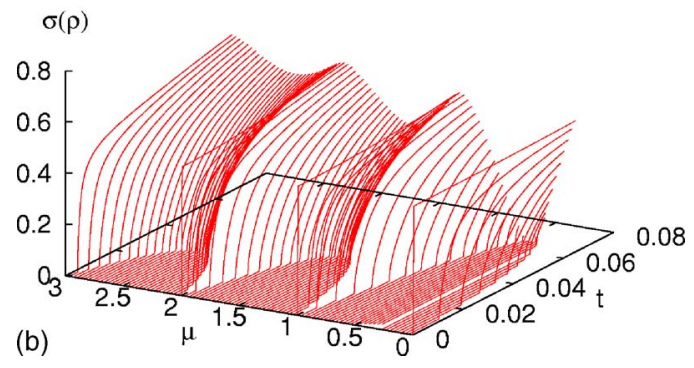

FIG. 2. (Color online) Phase diagram for the magnetic flux $\varphi$ $=0$ (right panel) and 0.4 (left panel). On the $z$ axis the variance $\sigma(\rho)$ of the Bose density $\rho$ is plotted. For the MI phase the variance of the $2 \mathrm{D}$ density is zero, and the Bose charge has no fluctuation.

stadter butterfly system ${ }^{22}$ to shrink, meaning that the superfluid order appears at a smaller value of $U$ (or higher value of t). For $\varphi \rightarrow 0.5$ the bandwidth of the Hofstadter butterfly is increased again and the critical value $t_{c}$ of the transition is increased. This suggests that in the phase diagram of Fig. 3 (calculated for $U=1$ ), the critical point $(U / t)_{c}$ of the SF-MI transition is proportional to the bandwidth of the Hofstadter butterfly. ${ }^{22}$

The loss of translational invariance of the charged bosons under a magnetic field is correlated with the appearance of the surface oscillations of the particle density in the superfluid phase. Although one expects a spontaneous breaking of the translational invariance in the superfluid phase, such as vortex states, in our mean-field approach this symmetry is explicitly broken by our gauge choice. Physically measurable quantities should be independent of the choice of the gauge, however our mean-field treatment yields spatially dependent parameters such as $\left|\Psi_{n}\right|^{2}$, which depend on the gauge choice. Still, it is not unreasonable to expect that spatially averaged quantities such as $\rho_{s}$ and magnetization to be correctly captured by our approach. For certain values of the magnetic field we verified this expectation by using the symmetric gauge and corresponding square supercell. Also, as the insulator side of the transition is spatially uniform, explicit determination of the gauge should not strongly affect the MI-superfluid phase boundaries. In Fig. 5 we show the variance of the on-site SF order parameter $\Psi_{n}$ when the hopping parameter $t$ is increased, for different points of the lattice $n$. Even all the SF order parameters $\Psi_{n}$ exhibit the disappearance of the insulator order at the same critical value, 

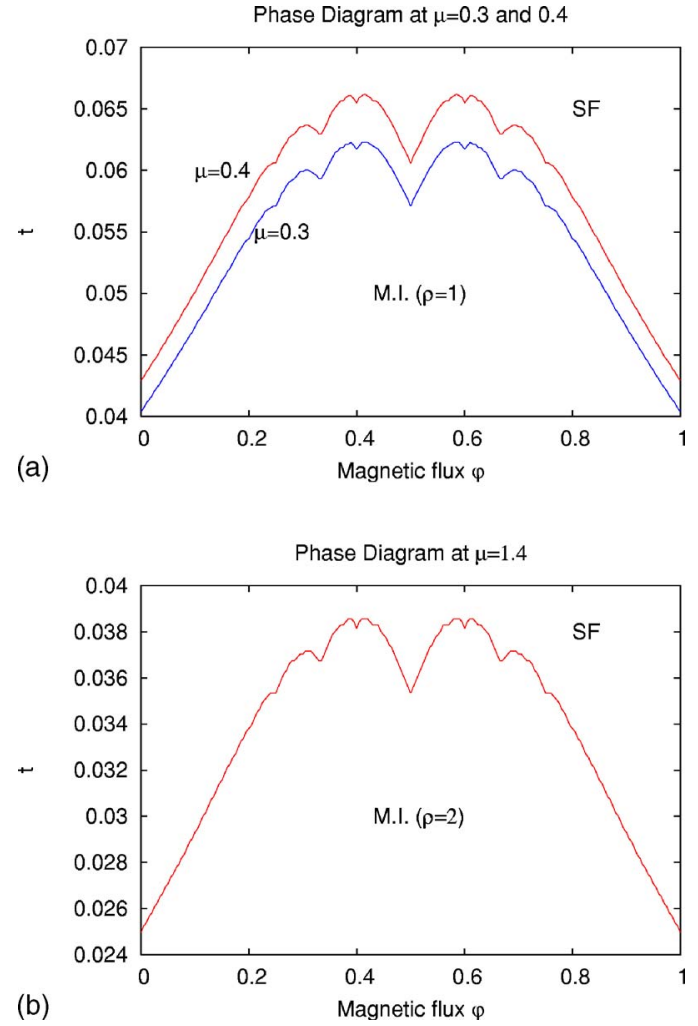

FIG. 3. (Color online) Phase diagram of Bose atoms confined in a $2 \mathrm{D}$ optical lattice in the $t-\varphi$ plane for different values of the chemical potential, corresponding to the MI phase with $\rho=1$ (top) and $\rho=2$ (bottom). The curves correspond to the critical values $t_{c}$ of the SF-MI transition. For $t>t_{c}$ the system is in the superfluid phase and for $t<t_{c}$ in the MI phase. The phase diagram is periodic in the magnetic flux with $\Delta \varphi=1$ and symmetric around the flux value $\varphi$ $=0.5$.

$t=t_{c}$, the rate of increase of $\Psi_{n}$ for $t>t_{c}$ is different for different points of the lattice. There are points in the lattice that show a very low rate of increase of $\Psi_{n}$. The figure clearly shows the surface oscillations of the "charge" density in the optical lattice. It means that, for $t>t_{c}$, there are regions in the lattice with small SF order parameter and small density fluctuations. It is possible to interpret these as the superfluid density oscillations due to the presence of vortices. However, close to the Mott transition where these regions are most pronounced, correlations between such regions may develop, causing a phase transition which would not be captured by our mean-field treatment.

The lower rate of increase of the superfluid order parameter vs $t$, for $t>t_{c}$, is also suggested in the scaled curves in Fig. 6 that show the ratio $\rho / \rho_{s}$ versus $t_{c} / t$. For $t_{c} / t \lesssim 1$, the SF phase is strongly affected by the magnetic field presence, and we note the change of the curve slope in Fig. 6 for $\varphi$ $\neq 0$. For $t_{c} / t \rightarrow 0$, the magnetic field has no effect, and all Bose particles tend to condense $\left(\rho / \rho_{s} \rightarrow 1\right.$ for all $\varphi$ values in Fig. 6). We also notice that even a small magnetic field $\varphi$ $=0.1$ affects the superfluid density rather strongly. For such small magnetic fields our approach is less reliable, as the cell size used in our calculations is inversely proportional to the flux $\varphi$. While similar discontinuous effects at zero field have

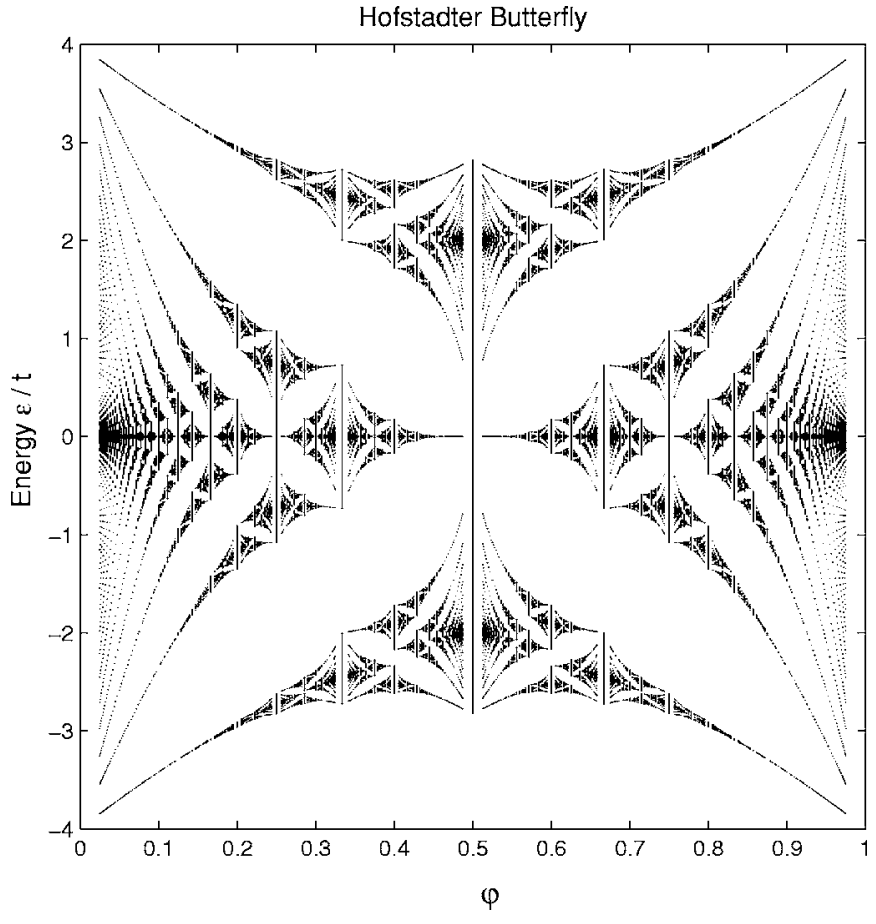

FIG. 4. Single particle energy bands as a function of magnetic flux per plaquette, the Hofstadter butterfly (Ref. 22).

been discussed within the context of Josephson junction arrays,${ }^{31}$ we cannot clarify the behavior near zero field due to the limitations of our numerical mean-field approach.

Figure 7 shows the magnetization as a function of the hopping parameter $t$ for different values of the magnetic flux $\varphi=0.1,0.2,0.3$, and 0.4 at the chemical potential $\mu=0.5$. We note that our approach is less reliable for the case of $\varphi$ $=0.1$, as the number of particles per site increase most quickly in this case. For $t<t_{c}$, in the MI phase, the magnetization is equal to zero and the particle motion is frozen. For $t>t_{c}$, in the SF phase, the curves exhibit negative values of magnetization, meaning the existence of persistent current flow. We note that the sign of the magnetization is related to the slope of the energy curve vs magnetic flux in Hofstadter butterfly. ${ }^{32}$

The change of slope of the eigenstates as a function of $\varphi$ in the Hofstadter butterfly gives rise to changes in the sign of the magnetization near the special values of the magnetic flux (for instance $\varphi=1 / q$; see Ref. 22). We do not expect this fine effect to be observable in the low $\varphi$ limit. In this case, the small fluctuations of the $\varphi$ for the real system gives rise to the smeared graph of the spectrum. ${ }^{22}$

For $\varphi \in[0.45,0.5]$ the magnetization can have an opposite sign (compared with the values for $\varphi \in[0,0.45]$ ) as the slope of the Hofstadter spectrum vs $\varphi$ clearly changes (see Ref. 22 or Fig. 3).

\section{SUMMARY}

We calculated the mean-field phase diagram of the $2 \mathrm{D}$ Bose-Hubbard Hamiltonian under a perpendicular magnetic 

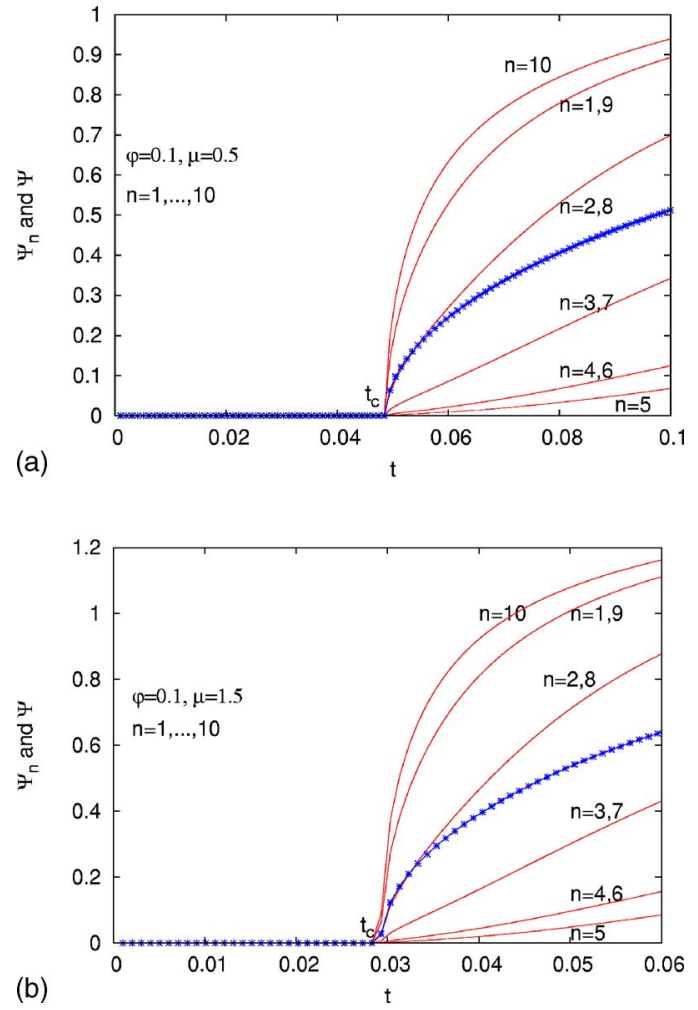

FIG. 5. (Color online) SF order parameter $\Psi_{n}$ for different lattice sites at MI-SF transition. The chemical potential is $\mu=0.5$ corresponding to MI with $\rho=1$ and $\mu=1.5$ corresponding to MI with $\rho=2$. The blue line with crosses represents the surface average value $\Psi$. For $t>t_{c}$ the second derivative $d^{2} \Psi / d t^{2}$ changes sign for the points $n=4,5$. The magnetic flux is $\varphi=0.1$ and the superfluid order parameter has the periodicity $\Psi_{n}=\Psi_{n+10}$. For the SF phase (at $t>t_{c}$ ) the lattice sites integer $\times 10+4$ and integer $\times 10+5$ exhibit very low values of the SF order parameter and low variance of $\rho$.

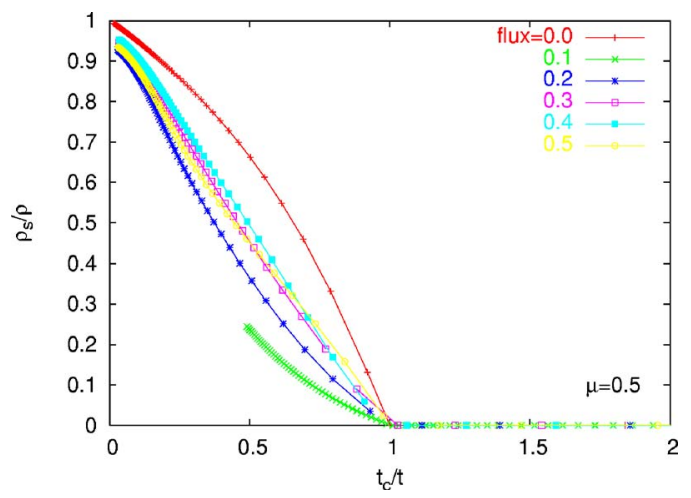

FIG. 6. (Color online) $\rho_{s} / \rho$ as a function of $t_{c} / t$ for different values of the magnetic flux. For $\varphi=0$ our curve is similar to Fig. 1 of Ref. 23. The change of slope at $t \simeq>t_{c}$ for $\varphi \neq 0$ is related to the surface oscillations of the charged bosons. When the magnetic field is present, the superfluid phase exhibits surface region where the quantum fluctuations of SF density and the order parameter are small (compared to nearby regions; see Fig. 5) meaning that the local phase is closer to an insulator. It exhibits a lower ratio $\rho_{s} / \rho$ compared with the case $\varphi=0$.

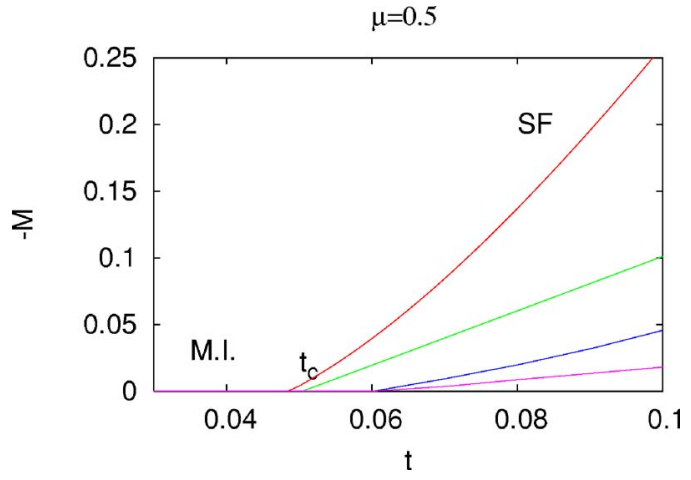

FIG. 7. (Color online) Magnetization as a function of $t$ for $\varphi$ $=0.1,0.2,0.3,0.4$ (from top to bottom). In the MI phase, for $t<t_{c}$, the magnetization is zero. Our numerical approach is more reliable at lower values of $t$, and not too small $\varphi$, where our computational supercell size is small and the number fluctuations at each lattice site are limited. MI and superfluid phases and $t_{c}$ are identified for $\varphi=0.1$ on the figure. The value of $t_{c}$ for other $\varphi$ can be identified as the point where magnetization becomes nonzero on the corresponding curve.

field. The Mott insulator-superfluid transition is strongly affected, and the features around the transition point resemble the interesting property of the Hofstadter butterfly. The energy spectrum for the noninteracting case exhibits periodicity with $\Delta \varphi=1$, symmetry around the value $\varphi=$ integer $/ 2$ and striking oscillations ${ }^{22}$ that lead to similar features of the MI-SF transition when the magnetic field is varied (see the phase diagram in the $t, \varphi$ plane in Fig. 3).

In the superfluid phase, at zero magnetic field the system has time translational invariance and the net local current of the Bose particles is zero because the reversed Bose paths are equally probable. Even for a small value of $\varphi$ the time invariance is suddenly broken and the reversal paths of the coherent Bose atoms are not equally probable anymore (in fact only one of the two reversal paths is permitted; the other one corresponds to change of the sign of the magnetic flux). The persistent currents of the Bose particles appear, leading to a nonzero value of the orbital magnetization. We note also the surface charge oscillations that are not present in the SF phase with translational invariance when the magnetic field is set to zero.

The surface charge oscillations are not present for the MI phase. At low values of $\varphi$ the SF phase exhibit negative susceptibility when the magnetic field is applied and at a critical value the system is driven into the insulating phase. This is the continuous limit of the model and the behavior of the Bose system shows features resembling that of the Meissner effect in superconductors. For higher kinetic energy (large values of $t / U$ ) this scenario is not valid anymore, the magnetic flux loses its effect over the quantum state of the Bose system and the system preserves its SF characteristics, albeit with spatial oscillations of its order paremeter. We hope our work stimulates further experimental and theoretical interest in this model. 


\section{ACKNOWLEDGMENTS}

M. N. acknowledges the support from TUBITAK and CERES and thanks the Department of Physics, Bilkent Uni- versity for hospitality during the time part of this work was performed. M.Ö.O is supported by TUBA-GEBIP Grant and TUBITAK-Kariyer Grant No. 104T165. B.T. gratefully acknowledges partial support from TUBITAK and TUBA.
*Electronic address: oktel@fen.bilkent.edu.tr

${ }^{1}$ G. Raithel, G. Birkl, A. Kastberg, W. D. Phillips, and S. L. Rolston, Phys. Rev. Lett. 78, 630 (1997); S. E. Hamann, D. L. Haycock, G. Klose, P. H. Pax, I. H. Deutsch, and P. S. Jessen, ibid. 80, 4149 (1998); L. Guidoni, C. Triché, P. Verkerk, and G. Grynberg, ibid. 79, 3363 (1997); M. Raizen, C. Salomon, and Q. Niu, Phys. Today 50(7), 30 (1997).

${ }^{2}$ F. Burgbacher and J. Audretsch, Phys. Rev. A 60, R3385 (1999).

${ }^{3}$ S. Inouye, A. P. Chikkatur, D. M. Stamper-Kurn, J. Stenger, D. E. Pritchard, and W. Ketterle, Science 285, 571 (1999).

${ }^{4}$ M. Kozuma, Y. Suzuki, Y. Torii, T. Sugiura, T. Kuga, E. W. Hagley, and L. Deng, Science 286, 2309 (1999).

${ }^{5}$ S. Inouye, T. Pfau, S. Gupta, A. P. Chikkatur, A. Görlit, D. E. Pritchard, and W. Ketterle, Nature (London) 402, 641 (1999).

${ }^{6}$ For a collection of review articles, see the special issue of Nature (London) 416, 206 (2002).

${ }^{7}$ E. Jane, G. Vidal, W. Dur, P. Zoller, and J. I. Cirac, Quantum Inf. Comput. 3, 15 (2003).

${ }^{8}$ O. Mandel, M. Greiner, A. Widera, T. Rom, T. W. Hänsch, and I. Bloch, Nature (London) 425, 937 (2003).

${ }^{9}$ W. K. Hensinger, H. Häffner, A. Browaeys, N. R. Heckenberg, K. Helmerson, C. McKenzie, G. J. Milburn, W. D. Phillips, S. L. Rolston, H. Rubinsztein-Dunlop, and B. Upcroft, Nature (London) 412, 52 (2001).

${ }^{10}$ C. M. Alves and D. Jaksch, Phys. Rev. Lett. 93, 110501 (2004).

${ }^{11}$ M. Greiner, O. Mandel, T. Esslinger, T. W. Hänsch, and I. Bloch, Nature (London) 415, 39 (2002); M. Greiner, O. Mandel, T. W. Hänsch and I. Bloch, ibid. 419, 51 (2002).

${ }^{12}$ B. Paredes, P. Zoller, and J. I. Cirac, Phys. Rev. A 66, 033609 (2002); N. Regnault and Th. Jolicoeur, Phys. Rev. Lett. 91, 030402 (2003).
${ }^{13}$ See for a recent review, D. Jaksch and P. Zoller, Ann. Phys. 315, 52 (2005).

${ }^{14}$ M. P. A. Fisher, P. B. Weichman, G. Grinstein, and D. S. Fisher, Phys. Rev. B 40, 546 (1998).

${ }^{15}$ D. Jaksch, C. Bruder, J. I. Cirac, C. W. Gardiner, and P. Zoller, Phys. Rev. Lett. 81, 3108 (1998), and references therein.

${ }^{16}$ D. Jaksch and P. Zoller, New J. Phys. 5, 56 (2003).

${ }^{17}$ A. S. Sorensen, E. Demler, and M. D. Lukin, Phys. Rev. Lett. 94, 086803 (2005).

${ }^{18}$ E. J. Mueller, Phys. Rev. A 70, 041603(R) (2004).

${ }^{19}$ S. Tung, V. Schweikhard, and E. A. Cornell, Phys. Rev. Lett. 97, 240402 (2006).

${ }^{20}$ K. Osterloh, M. Baig, L. Santos, P. Zoller, and M. Lewenstein, Phys. Rev. Lett. 95, 010403 (2005).

${ }^{21}$ J. Ruseckas, G. Juzeliunas, P. Ohberg, and M. Fleischhauer, Phys. Rev. Lett. 95, 010404 (2005).

${ }^{22}$ D. R. Hofstadter, Phys. Rev. B 14, 2239 (1976).

${ }^{23}$ K. Sheshadri, H. R. Krishnamurthy, R. Pandit, and T. V. Ramakrishnan, Europhys. Lett. 22, 257 (1993).

${ }^{24}$ M. Niemeyer, J. K. Freericks, and H. Monien, Phys. Rev. B 60, 2357 (1999).

${ }^{25}$ J. K. Freericks, and H. Monien, Phys. Rev. B 53, 2691 (1996).

${ }^{26}$ P. Buonsante and A. Vezzani, Phys. Rev. A 70, 033608 (2004).

${ }^{27}$ P. Buonsante and A. Vezzani, Phys. Rev. A 72, 013614 (2005).

${ }^{28}$ D. van Oosten, P. van der Straten, and H. T. C. Stoof, Phys. Rev. A 63, 053601 (2003).

${ }^{29}$ G. Bergmann, Phys. Rep. 207, 1 (1984).

${ }^{30}$ M. Tinkham, Superconductivity (McGraw-Hill, New York, 1996).

${ }^{31}$ Q. Niu and F. Nori, Phys. Rev. B 39, 2134 (1989).

${ }^{32}$ M. Niță, A. Aldea, and J. Zittartz, Phys. Rev. B 62, 15367 (2000). 